\title{
The Influence of Spokesperson Trustworthiness on Message Elaboration, Attitude Strength, and Advertising Effectiveness
}

\author{
Joseph R. Priester \\ University of Michigan \\ Richard E. Petty \\ Ohio State University
}

\begin{abstract}
Recent research has shown that information presented by untrustworthy endorsers is likely to be thoughtfully elaborated, whereas information presented by trustworthy endorsers is likely to be unthinkingly accepted (Priester \& Petty, 1995). Study 1 manipulated argument quality and assessed cognitive responses to demonstrate that this influence of trustworthiness on persuasion holds for familiar endorsers likely to be used in actual advertisements. Study 2 demonstrated that trustworthiness can be influenced by individuals endorsing too many products, with similar persuasion consequences (i.e., untrustworthy endorsers prompt greater scrutiny of product-related attributes than trustworthy endorsers). Study 2 also found that the attitudes that resulted from an untrustworthy endorser came to mind faster, even when those attitudes were equivalently positive - thus demonstrating that elaboration can influence attitude accessibility. This research provides evidence that attitude extremity may not be a sufficient indicator of advertising effectiveness, in that equally extreme attitudes can vary as to the bases by which they were formed, their underlying strength, and their effectiveness. Further, this research provides theoretical and strategic insight into the use of trustworthy and untrustworthy endorsers.
\end{abstract}

The goal of advertising is to present information to potential customers. This information, it is hoped, will result in customers adopting more favorable attitudes toward the advertised product or service. These attitudes, in turn, should result in a greater probability of the customer purchasing the advertised product or using the service than if the customer had not been exposed to the advertisement. ${ }^{1}$ A common strategy in advertising is to have an endorser present information. But what attributes are desirable for an endorser to possess? This question is particularly important given research suggesting that the process by which an attitude is formed or changed has crucial implications for the effectiveness of that attitude to guide future behavior, such as purchasing a product (see Petty, Priester, \& Wegener, 1994; Petty \& Wegener, 1997; Priester \& Fleming, 1997; see also Priester, Nayakan Kuppam, Fleming, \& Godek, in press). The goal of this article is to examine how endorser

Requests for reprints should be sent to Joseph R. Priester, University of Michigan Business School, 701 Tappan Street, Ann Arbor, MI 48109-1234. E-mail: priester@umich.edu

${ }^{1}$ Of course, there are times that advertising is intended not to persuade, but instead to remind consumers of a product. trustworthiness influences message elaboration and advertising effectiveness. A trustworthy endorser is one whom people perceive to be honest and sincere, whereas an untrustworthy endorser is one about whom people feel skepticism and suspicion (i.e., people question whether one can be trusted to communicate the truth). ${ }^{2}$

Contemporary theories in attitudes and persuasion have introduced the notion that persuasion can be the result of qualitatively different processes. That is, the Elaboration

\footnotetext{
${ }^{2}$ It should be noted that the variable of interest here is trustworthiness, not credibility. Since at least 1953, communicator effectiveness has been conceptualized within the general construct of credibility. The specific components most often hypothesized to constitute credibility are expertise and trustworthiness (Hovland, Janis, \& Kelley, 1953). Expertise refers to a communicator's ability to confer accurate information (i.e., an expert source possesses the requisite knowledge necessary to provide accurate information). Trustworthiness refers to a communicator's intent to transmit accurate information (i.e., a trustworthy source is believed to be honest rather than deceptive in communicating information; see Hovland et al., 1953, Chap. 2, for further discussion). Our research seeks to examine the implications of endorser trustworthiness on message processing and persuasion while holding endorser expertise constant at a high level.
} 
Likelihood Model of persuasion (ELM; Petty \& Cacioppo, 1981, 1986) and the Heuristic-Systematic Model (HSM; Chaiken, Liberman, \& Eagly, 1989) contend that attitudes can be changed following either a careful and effortful scrutiny of a message (elaboration-based process) or less cognitively effortful inference and associative processes. In general, research has revealed that persuasion is more likely to be the result of thoughtful message elaboration when individuals are both motivated and able to consider the information. In contrast, when individuals lack either the motivation or ability to consider the merits of the arguments, they rely instead on relatively less thoughtful processes (for recent reviews, see Chaiken, Wood, \& Eagly, 1996; Petty et al., 1994; Petty \& Wegener, 1997).

\section{TRUSTWORTHINESS AND ELABORATION}

Recent research has suggested that endorser trustworthiness can influence persuasion by affecting the likelihood that recipients will engage in message-based elaboration under conditions of moderate elaboration likelihood. Specifically, Priester and Petty (1995) argued that given a situation in which individuals possessed the ability, but lacked the motivation to elaborate an advertisement, such as when the message is of low personal relevance (Petty \& Cacioppo, 1979) or the message recipients do not enjoy thinking (i.e., low in need for cognition; Cacioppo \& Petty, 1982), there would be a greater need to think about a message from an expert source who was low rather than high in trustworthiness. That is, if a message recipient can be confident that an expert source will be willing to provide accurate information because of his or her high trustworthiness, they may forgo the effortful task of scrutinizing the message and, instead, unthinkingly accept the conclusion as valid. In contrast, if a message recipient is unsure as to whether an expert source will provide accurate information because of the source's low or questionable trustworthiness, he or she may feel the need to scrutinize the arguments to ascertain if the communication is indeed cogent and valid. Thus, this perspective suggests that endorsers low in trustworthiness can influence attitudes by increasing the amount of attention paid to the advertisement.

This finding is particularly surprising given the widespread belief that information associated with an untrustworthy endorser will most likely be unthinkingly rejected, or at the very least, severely devalued. And perhaps the most provocative implications of the Priester and Petty results arise when the conclusions are generalized to the field of advertising. The results suggest that advertisers might sometimes behoove themselves to utilize endorsers of low or questionable trustworthiness. Obviously, before such a counterintuitive conclusion is advanced, additional research is warranted.

Perhaps the most important question bears on the generalizability of the Priester and Petty results. In all of the Priester and Petty experiments, hypothetical sources were used for when the message recipients had no information other than that provided in the experiment. In addition, the cover story used in these experiments asked the participants to form an impression of the speaker. Taken together, these two observations provide the basis for an alternative explanation to the Priester and Petty results such that message recipients elaborated information from an untrustworthy more than a trustworthy endorser to come to a more confident impression about the unfamiliar source. If such an alternative explanation is sound, untrustworthy endorsers with whom the audiences are familiar should not lead to greater message elaboration than familiar trustworthy endorsers. And the implication that untrustworthy endorsers can sometimes be more effective than trustworthy endorsers would not be warranted. Thus, a critical question arises as to whether the influence of trustworthiness on message elaboration will be observed when the endorsers are individuals with whom the audience is familiar before the experiment. Experiment 1 was conducted to address this question directly by using endorsers with whom the audience was familiar.

\section{INDICATORS OF ELABORATION}

One method commonly used to investigate the impact of variables on the extent of elaboration is the measurement of cognitive responses (Greenwald, 1968; Petty, Ostrom, \& Brock, 1981; Wright, 1973, 1980). In this approach, both the attitude that results from a persuasive communication and the thoughts that occurred to a message recipient during exposure to the persuasive communication are assessed. Differences in the extent of message elaboration are inferred by differences in the number of thoughts generated or in the extent to which these thoughts predict the resulting attitude. ${ }^{3}$ In response to criticisms concerning the cognitive response approach, a manipulation of argument quality was introduced as an alternative means of assessing the extent of message scrutiny (Petty, Wells, \& Brock, 1976; see Petty \& Cacioppo, 1986, pp. 30-44; Petty, Wegener, Fabrigar, Priester, \& Cacioppo, 1993). The logic of manipulating argument quality to assess the extent of thinking is that when individuals are exposed to a message under specific conditions that foster message-based elaboration, the quality of the arguments should have a larger impact on attitudes than when elaboration is low. That is, the greater influence of argument quality

\footnotetext{
${ }^{3}$ Although the cognitive response approach is widely used, it is not without criticism (see Eagly \& Chaiken, 1993, pp. 293-296; Miller \& Coleman, 1981). Much of this criticism focuses on the correlational nature of the approach. Specifically, researchers using the cognitive response approach often wish to make the inference that, under specific conditions, thoughts are the result of effortful elaboration during the message presentation and these thoughts are leading to attitudes. However, given the correlational nature of this approach, it is alternatively possible that, under specific conditions, the reported attitudes are leading to thoughts.
} 
on attitudes for one level of a variable than another provides an inference of differences in the extent to which individuals are basing their attitudes on a thoughtful elaboration of the arguments in the advertisement (see Petty \& Cacioppo, 1986).

\section{EXPERIMENT 1}

To examine whether the influence of endorser trustworthiness on elaboration found in Priester and Petty (1995) generalizes to familiar endorsers, an advertisement was constructed that used familiar endorsers of either high of low trustworthiness. To examine differences in product-related elaboration as a function of endorser trustworthiness, both the cognitive response and the manipulation of argument quality approaches were used. Both approaches were used to (a) provide convergent evidence of differences in product-related elaboration as a function of endorser trustworthiness and (b) provide mediational evidence of thoughtful versus nonthoughtful attitude change.

\section{Hypotheses}

Thus, the experiment manipulated Argument Quality and Endorser Trustworthiness. Our key hypothesis (H1) was that an Endorser Trustworthiness $\times$ Argument Quality interaction would emerge on the measure of attitude toward the product. This interaction would reveal that argument quality has a larger impact on peoples' attitudes when the endorser was low rather than high in trustworthiness ( $\mathrm{H} 1 \mathrm{a})$. In addition, we hypothesized that attitudes resulting from a high and low trustworthy endorser would differ in terms of their bases (H2). Specifically, the attitudes should be more highly correlated with the valence of product-relevant cognitive responses when the endorser was low rather than high in trustworthiness ( $\mathrm{H} 2 \mathrm{a})$. In contrast, we hypothesized that, if anything, the attitudes should be more highly correlated with the general positive perception of the endorser when the endorser was high rather than low in trustworthiness $(\mathrm{H} 2 \mathrm{~b})$. This prediction stems from the hypothesis that individuals are more likely to unthinkingly accept the position of a high trustworthy source, and would be more accepting of the message the more trustworthy they found the source to be. This configuration of attitude and cognitive response results would provide evidence that prior results do extend to familiar sources.

\section{Method}

\section{Procedure}

A total of 65 undergraduate students at the Ohio State University participated. The students were randomly assigned to one of 4 conditions in a $2 \times 2$ (Endorser Trustwor- thiness: High or Low $\times$ Argument Quality: Strong or Weak) factorial experiment. Experimental sessions were conducted in groups of 6 to 10 .

All participants received an experimental booklet entitled "Athletics and Advertising." The first page of the booklet explained that there had been a recent trend in advertising that integrated athletics with advertising. All participants were informed that the booklets contained three advertisements that reflected this trend and that they should read the three advertisements as if they came across them in a magazine. They were further told that their overall reactions to the advertisements would be assessed at the end of the booklet.

One advertisement appeared on each of the second, third, and fourth pages of the booklet. All of these ads were presented in black and white, with comparable images. The first and third advertisements were taken from popular running magazines. They were advertisements for a nutritional bar and running pants. The second advertisement was the target advertisement and is described following. After reading the three advertisements, participants were informed that because of time constraints, they would only be asked about the second of the three advertisements (i.e., the target advertisement). Participants then answered a series of questions and provided their cognitive responses to the advertisement. After all participants in a group had completed the experimental booklets, they were debriefed and excused.

\section{Independent Variables}

Endorser trustworthiness. Endorser trustworthiness was manipulated by using either Nancy Kerrigan (high trustworthiness) or Tonya Harding (low trustworthiness) as the endorser of a fictitious product-Mercury Roller-Blades. ${ }^{4}$ The study was conducted in 1994 at the start of the Winter Olympic Games. At the time the experiment was conducted, there was a great deal of media attention devoted to the two skaters. Although both skaters possessed expertise about the product, Nancy Kerrigan was generally viewed as a relatively trustworthy person, whereas Tonya Harding was generally viewed as a relatively untrustworthy person. Thus, these individuals were used to instantiate endorsers of high (Nancy Kerrigan) and low (Tonya Harding) trustworthiness. A picture of the endorser appeared in the upper left corner of the advertisement. To the right of the picture appeared the endorser's name, under which appeared "U.S. Olympic Representative."

Argument quality. Strong and weak arguments were developed and pretested (see Petty \& Cacioppo, 1986) in

\footnotetext{
${ }^{4}$ Roller-blades were chosen as the product because both endorsers, due to their expertise in figure skating, could be expected to have relatively high and comparable expertise toward the product. The product was also chosen because of its interest to the target population in our sample.
} 
support of the fictitious product-Mercury Roller-Blades. ${ }^{5}$ Both strong and weak arguments were constructed such that they described five attributes of the product. These attributes included the fit, cushioning, safety, available colors, and price of the roller-blades. The text of the strong and weak arguments are included in the appendix. ${ }^{6}$

\section{Dependent Variables}

Manipulation checks. Participants answered questions designed to serve as manipulation checks on the Argument Quality and Endorser Trustworthiness manipulations. To assess perceived argument quality, participants were asked to rate how strong the reasons used in the advertisement were on a scale ranging from -4 (very weak) and +4 (very strong). To assess perceived endorser trustworthiness, participants were instructed that "because your impressions of the advertisement may have been influenced by your feelings for Nancy Kerrigan (or Tonya Harding; whichever endorser they were exposed to), we would like you to tell us how you feel about (the endorser) by circling whichever number you feel most appropriate on the line below." Imbedded within 10 endorser attributes was trustworthiness. Participants provided their rating of trustworthiness on an 11 -point scale ranging from 1 (untrustworthy) to 11 (trustworthy).

Attitude measures. On the third page of the questionnaire, participants were asked to provide responses indicative of their attitudes toward the advertised product using a 5-item semantic differential. The participants were asked to "rate how you feel about the Mercury Roller-Blades by circling a number on each of the lines below." Each semantic differential was on a 9-point scale ranging from -4 (negative, harm-

\footnotetext{
${ }^{5}$ It is important to reiterate the rationale for the use of the argument quality manipulation. In our experiment, half of the participants receive advertisements specifically designed to elicit unfavorable cognitive responses. This manipulation is used as a methodological tool to provide data from which to make inferences about the processes underlying attitude change. The value of using the manipulation of argument quality is that it allows insight into how individuals respond to the information provided by endorsers of low and high trustworthiness. Obviously, one would never deliberately present advertisements comprised of purposefully weak information (i.e., weak arguments) in a promotion campaign. Nevertheless, the use of an argument quality manipulation in pretesting can provide insight into whether recipients are paying attention to the substantive information presented or not.

${ }^{6}$ As part of a separate experiment conducted to rule out alternative hypotheses, 37 people were asked to rate the arguments. Three separate questions were used to indicate the ease or difficulty of understanding the arguments. These questions were presented on 9-point scales ranging from -4 (difficult to understand, complex, and difficult to think about) to +4 (easy to understand, simple, and easy to think about). The alpha coefficient for these three items was .97; thus, the three questions were averaged to create one measure. An Argument Quality $\times$ Endorser Trustworthiness analysis of variance revealed no differences in the ease/difficulty of the arguments as a function of argument quality, $F(1,33)<.2, p>.6$. Thus, any differences in persuasion as a function of Argument Quality could not be explained by differences in ease of processing of the strong and weak arguments.
}

ful, foolish, bad, and unfavorable) to +4 (positive, beneficial, wise, good, and favorable).

Cognitive responses. After completing the attitude measures, individuals were asked to list the thoughts that came to mind while reading the message. Participants were provided a page containing eight boxes and were instructed to write their thoughts in these boxes-one thought per box. Participants were further instructed to use only as many boxes as the number of thoughts that they could recall (see Petty \& Cacioppo, 1986). Participants read that they should spend at least 3 , but no more than 10 min listing their thoughts. After listing their thoughts, the participants were instructed to code each of the listed thoughts along two dimensions. First, they were asked to identify each thought as to its evaluative valence coding each thought as positive, negative, or neutral/unrelated to the advertisement. Second, participants were asked to identify each thought as to its subject matter. Participants coded each thought as focused on the product in the advertisement, the spokesperson associated with the topic, or neither.

\section{Results}

\section{Manipulation Checks}

The manipulation checks were subjected to $2 \times 2$ (Endorser Trustworthiness $\times$ Argument Quality) analysis of variance. As expected, participants who read the advertisement comprised of strong arguments rated the reasons used in the advertisement as stronger $(M=1.1)$ then those participants who read the advertisement comprised of weak arguments $(M=-.9), F(1,61)=28.2, p<.0001$.

Also as predicted, participants perceived Nancy Kerrigan to be considerably more trustworthy $(M=7.2)$ than Tonya Harding $(M=3.3), F(1,61)=68.2, p<.0001$. Although not manipulated, it should be noted that participants were also asked to rate the extent to which the endorser was knowledgeable about roller-blading. On this measure there were no statistically significant effects for either Endorser Trustworthiness or Argument Quality. In summary, the endorser perception measures found that using the two figure skaters as instantiations of two equal experts who differed in trustworthiness was effective.

\section{Attitude Measures}

The five semantical differential items were averaged to create an attitude index. The standardized alpha coefficient for this index was .96 . The attitude index was analyzed by a 2 $\times 2$ (Endorser Trustworthiness $\times$ Argument Quality) analysis of variance. The means and standard deviations for the four experimental conditions are presented in Table 1 and graphed in Figure 1.

Of greatest importance, there emerged the predicted Endorser Trustworthiness $\times$ Argument Quality interaction, $F(1$, 
TABLE 1

Means, Standard Deviations, and Cell Numbers for Attitude Index, Experiment 1

\begin{tabular}{lcccccc}
\hline & \multicolumn{2}{c}{ Tonya Harding } & & \multicolumn{2}{c}{ Nancy Kerrigan } \\
\cline { 2 - 3 } \cline { 6 - 7 } & $M$ & $S D$ & & $M$ & $S D$ \\
\hline Strong arguments & $1.2^{\mathrm{a}}$ & 1.0 & & $1.2^{\mathrm{a}}$ & 0.8 \\
Weak arguments & $-1.0^{\mathrm{a}}$ & 1.8 & & $0.5^{\mathrm{b}}$ & 1.0 \\
\hline
\end{tabular}

$\mathrm{a}_{n}=16 ; \mathrm{b}_{n}=17$.

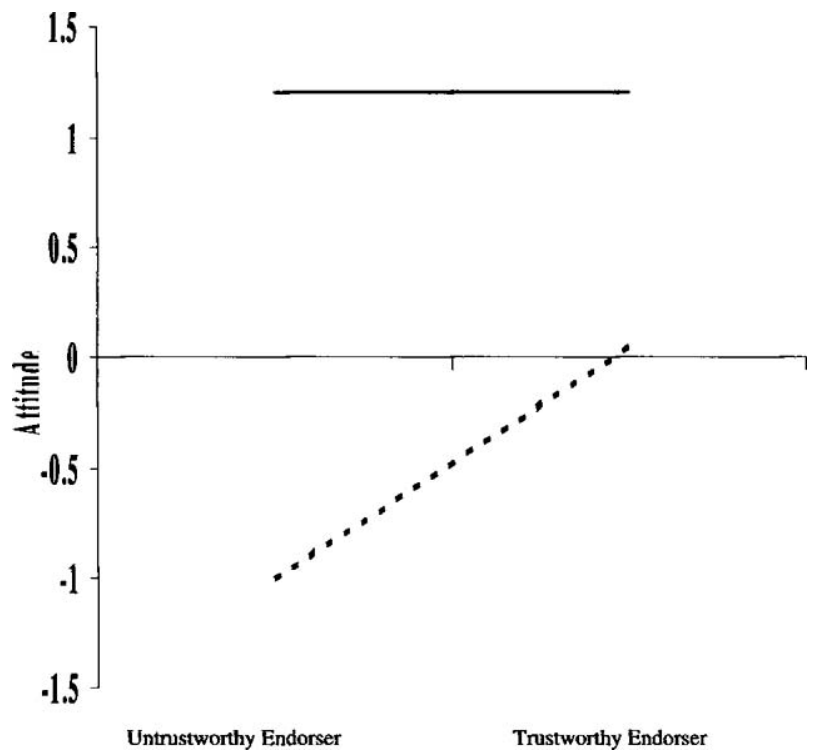

FIGURE 1 Attitude as a function of endorser and argument quality: Experiment 1.

$61)=6.7, p<.02$-providing support for hypothesis $\mathrm{H} 1 .^{7}$ This interaction revealed that participants who read the advertisement endorsed by Tonya Harding were more influenced by Argument Quality, $F(1,31)=18.1, p<.0002$, than were participants who read the advertisement endorsed by Nancy Kerrigan, $F(1,32)=4.1, p<.06$. That is, the attitudes of the participants exposed to the Tonya Harding endorsement were more influenced by strong $(M=1.2)$ than weak ( $M$ $=-1.0)$ arguments to a greater extent than the participants exposed to the Nancy Kerrigan endorsement ( $M=1.2$ for strong arguments, $M=.5$ for weak arguments). Thus, the attitude indexes supported the hypothesis H1a that an endorser of low trustworthiness can lead to greater scrutiny of product-related information than an endorser of high trustworthiness.

\footnotetext{
${ }^{7}$ Results also revealed significant main effects for both Trustworthiness and Argument Quality. First, there was a significant main effect for Endorser Trustworthiness, $F(1,61)=6.9, p<.02$. Participants had more favorable attitudes toward the product when it was endorsed by Nancy Kerrigan $(M=.8)$ than by Tonya Harding $(M=.1)$. Second, there was a main effect for Argument Quality, $F(1,61)=22.2, p<.0001$. Not surprisingly, participants were more persuaded by the advertisement containing strong $(M=1.2)$ rather than weak $(M=-.2)$ arguments.
}

It is worth commenting on the specific form of the Endorser Trustworthiness $\times$ Argument Quality interaction. Specifically, the attitudes for participants who read a strong argument advertisement endorsed by Nancy Kerrigan were as persuaded as the participants who read an advertisement endorsed by Tonya Harding. However, it should be recalled that the bases of these attitudes were hypothesized to differ. Specifically, participants were predicted to have adopted favorable attitudes following the ad by Tonya Harding largely because elaboration of the strong arguments resulted in favorable reactions to those arguments. However, people were relatively favorable toward the product endorsed by Nancy Kerrigan regardless of argument quality. Because participants exposed to the Nancy Kerrigan ad did not base their attitudes as much on argument quality, it is most likely that the favorable attitudes engendered by the Nancy Kerrigan endorsement were the result of a relatively low-effort process such as deciding to like the skates because of the positive image of Nancy Kerrigan rather than because of favorable thoughts about the actual merits of the skates. Evidence supportive of this interpretation is presented next.

\section{Attitude Predictors}

A product-related cognitive response ( $\mathrm{PCR}$ ) index was created by subtracting the product focused negative from the product focused positive cognitive responses. This measure thus provided an indication of the overall valence of the thoughts generated about the product from the information in the advertisement (see Cacioppo, Harkins, \& Petty, 1981). If participants' attitudes were based more on product-related elaboration when the endorser was Tonya Harding than Nancy Kerrigan, participants' attitudes should be more highly correlated with their PCRs in the latter than in the former conditions. To examine this prediction, the PCR index was correlated with the postmessage attitude index for each of the endorsers. ${ }^{8}$ The results of these correlational analyses reveal that for participants exposed to the advertisement endorsed by Tonya Harding, the correlation between the PCR and the attitude index is significant $(r=.60)$, whereas for the participants exposed to the advertisement endorsed by Nancy Kerrigan, this correlation $(r=.25)$ is not statistically significant.

For those participants exposed to an ad featuring Nancy Kerrigan, attitudes were expected to be based on how positively participants perceived the source. To examine this pre-

\footnotetext{
${ }^{8}$ Cognitive response to attitude correlations has been used quite often to provide an inference of elaborative processing (e.g., Chaiken, 1980; Mackie, 1987; Petty \& Cacioppo, 1979; Priester \& Petty, 1995). Such an approach presumes that the cognitive response profiles (e.g., the positive minus negative responses) predict attitudes differentially as a function of elaboration. Thus, although individuals may generate equivalent numbers of cognitive responses in response to the thought listing task, it is the extent to which these cognitive responses influence an individual's attitude that determines the extent to which his or her evaluation is based on thoughtful consideration versus nonthoughtful acceptance of the advertisement information.
} 
diction, attitudes were correlated with the measure of perceived trustworthiness for each of the endorsers. The results revealed that the correlation between perceived endorser trustworthiness and the attitude index is significant when the endorser is Nancy Kerrigan $(r=.43)$, but is not statistically significant when the endorser is Tonya Harding $(r=.11)$.

To test whether the correlations differed significantly from each other, Fisher's $r$ to $z$ transformations were used. In support of hypothesis $\mathrm{H} 2 \mathrm{a}$, analyses revealed that attitudes were significantly based more on product-focused thoughts when the endorser was Tonya Harding than Nancy Kerrigan $(z=1.80, p<.05)$, and in support of hypothesis $\mathrm{H} 2 \mathrm{~b}$, attitudes were based marginally more on perceived trustworthiness when the endorser was Nancy Kerrigan than Tonya Harding $(z=1.34, p<.1)$. Thus, the correlations between the attitude measure and the attitude predictors were consistent with hypothesis $\mathrm{H} 2$ - that the attitudes resulting from a high and low trustworthy endorser would differ in terms of their bases. Specifically, the advertisement featuring an endorsement by a low trustworthy endorser (i.e., Tonya Harding) produced attitudes that were based on PCRs to a greater extent than the advertisement featuring an endorsement by a high trustworthy endorser (i.e., Nancy Kerrigan). In addition, the correlations suggested that the product endorsed by the high trustworthy endorser (i.e., Nancy Kerrigan) produced attitudes that were based more on a simple cue inference process (perception of trustworthiness) than the product endorsed by the low trustworthy endorser (i.e., Tonya Harding).

Clearly, one would never use weak arguments for an actual advertisement. Rather, argument quality was manipulated in this experiment to provide evidence of differences in elaboration of product-related information. And the attitude mediation results, collapsed across strong and weak arguments, are consistent with the hypotheses. However, these analyses do not specifically address the question of the equivalent attitudes formed for each endorser who presented strong arguments. Although the attitudes are equivalent as assessed by attitude extremity, they are hypothesized to have been formed by different psychological processes.

To examine this question in a more focused manner, the correlations between the attitudes and attitude predictors were analyzed as a function of endorser for only those participants who received the strong arguments. As predicted, these correlational analyses revealed that the general mediational pattern held even for those participants exposed to the strong arguments: For the people exposed to the advertisement containing strong arguments endorsed by Tonya Harding, the correlation between the PCR and the attitude index is significant $(r=.62)$, whereas for the participants exposed to the advertisement containing strong arguments endorsed by Nancy Kerrigan, this correlation ( $r=$ .08 ) is not statistically significant. That is, although the attitudes resulting from both endorsers were equally positive (given strong arguments), the attitudes did differ in their bases.

\section{Conclusions}

Recall that Experiment 1 was conducted to examine whether the finding that familiar untrustworthy endorsers results in greater elaboration than familiar trustworthy endorsers. Both the significant Argument Quality $\times$ Trustworthiness interaction on the attitude measure, as well as the attitude predictor correlation results, support this generalization. Specifically, the results were consistent with the view that (a) endorsers who are low in trustworthiness engender greater elaboration of the product-related information contained in the advertisement than endorsers who are high in trustworthiness and (b) endorsers who are high in trustworthiness engender a relatively nonthoughtful acceptance — even when those endorsers are familiar.

\section{Emerging Questions}

The results of Experiment 1 provide support for the hypothesis that familiar endorsers low in trustworthiness can lead to greater elaboration of product-relevant information than endorsers high in trustworthiness. Yet several questions emerge from the findings of Experiment 1.

Elaboration interpretation of the attitude interaction. It should be recalled that our conclusions were inferred from the predicted Trustworthiness $\times$ Argument Quality interaction and the correlations obtained between the brand attitudes and the attitude predictors (i.e., PCR index and perceptions of trustworthiness). Specifically, we interpreted the nature of the attitude interaction by examining the influence of argument quality separately for the high and low trustworthiness endorsers. This interpretation suggests that argument quality influences the attitudes of participants exposed to an advertisement endorsed by an untrustworthy more than a trustworthy source.

As noted earlier, inspection of Figure 1 reveals the finding that for the strong argument conditions, trustworthy and untrustworthy endorsers resulted in equivalently positive attitudes. Our explanation of these equivalently extreme attitudes is that the psychological processes by which these attitudes were formed (i.e., the bases) differ. Similar results have been found in research examining the influence of mood on persuasion. For example, in two experiments, Petty, Schumann, Richman, and Strathman (1993) found that positive mood led to greater attitude change than neutral mood. However, mediational analyses revealed that when individuals were motivated to think about the merits of the message, mood influenced the proportion of positive beliefs and beliefs in turn influenced attitudes. In contrast, when participants were not motivated to think about the merits of the information, mood influenced attitudes without influencing beliefs. That is, mood influenced attitudes either by a thoughtful or a nonthoughtful process. Similarly, we argue that endorser trustworthiness, when paired with strong arguments, led to favorable attitudes both by a thoughtful and a nonthoughtful process. 
Alternative interpretation of the attitude interaction. There exists a different interpretation of the attitude interaction. Specifically, one can examine the influence of trustworthiness separately for strong and weak arguments. Such an examination reveals that Nancy Kerrigan produces more favorable attitudes than Tonya Harding in the weak argument condition and there is no effect in the strong argument condition. One might infer from this interpretation of the interaction that the conclusion that an untrustworthy endorser is superior is not supported. ${ }^{9}$ It should be noted that such an interpretation relies on attitude extremity as the key measure of advertising effectiveness. To wit, this interpretation argues that because the attitudes resulting from the trustworthy and untrustworthy endorsers are equally positive, there is no superiority of the untrustworthy endorser.

Elaboration and attitude strength. Given these two, quite different interpretations of the data, a critical question arises as to whether it is possible to demonstrate clearly that the attitudes resulting from advertisements associated with untrustworthy endorsers are more effective than the attitudes resulting from advertisements associated with trustworthy endorsers, even when those attitudes are equally positive. Past research on the influence of elaboration on attitude strength has provided evidence that attitudes that are the result of elaboration are stronger than attitudes that are based on less thoughtful persuasion processes (see Petty \& Cacioppo, 1986; Petty, Haugtvedt, \& Smith, 1995; Petty et al., 1994, for reviews). For example, attitudes based on elaboration have been found to persist longer than nonthoughtful attitudes (e.g., Chaiken, 1980; Elms, 1966; Haugtvedt \& Petty, 1992; Verplanken, 1991). Similarly, elaborated attitudes have been found to be more resistant to counter-persuasion than nonthoughtful attitudes (e.g., Haugtvedt \& Petty, 1992; Haugtvedt \& Wegener, 1994). And perhaps most important, attitudes that are the result of elaboration have been found to be more predictive of behavior than nonthoughtful attitudes (e.g., Cacioppo, Petty, Kao, \& Rodriquez, 1986; Kallgren \& Wood, 1986; Leippe \& Elkin, 1987; Verplanken, 1991).

Research has also provided extensive support for the notion that the speed at which an evaluation comes to mind (i.e., the accessibility of an attitude) is an index of the strength of that attitude (e.g., Fazio, 1995). And prior work has suggested a theoretically cogent link between elaboration and accessibility. Specifically, Rennier (1988, reported in Petty et al., 1995) found that attitudes that were changed as a result of relatively thoughtful processes were more accessible-came to mind faster-than attitudes that were changed as a result of relatively nonthoughtful processes. ${ }^{10}$ These various re-

\footnotetext{
${ }^{9}$ Such an interpretation would also need to account for the differences in the correlation pattern among the attitudes predictors and the attitude, of course.

10Interestingly, research also suggests that in addition, attitude accessibility can influence message elaboration (Fabrigar, Priester, Petty, \& Wegener 1998).
}

search findings suggest that a theoretically meaningful indicator of attitude strength is the speed with which an attitude comes to mind.

Tests of competing interpretations. Although Experiment 1 provided evidence in support of the prediction that endorsers low rather than high in trustworthiness can lead to greater elaboration of product-related information, it did not provide direct evidence that the attitudes resulting from an untrustworthy endorser are stronger than the attitudes resulting from a trustworthy endorser. Such a finding would provide compelling support for the elaboration, over the alternative, attitude interaction interpretation. In addition, such a finding would constitute a contribution above and beyond the findings of Petty et al. (1993). Recall that Petty et al. (1993) provided evidence that equivalently extreme attitudes could be the result of different psychological processes. However, this evidence was in the form of attitude to cognitive response correlations and did not provide evidence of the differential effectiveness of the attitudes. Differences in attitude accessibility would provide such unique evidence. In fact, although the Rennier (1988) finding is widely reported, the finding that elaboration leads to greater accessibility would represent the first published reporting of this relation.

Manipulation of trustworthiness. It is clear that the instantiations of trustworthiness used in Experiment 1 (i.e., Tonya Harding and Nancy Kerrigan) are associated with attributes other than trustworthiness upon which these individuals also differed. That is, these individuals were chosen to manipulate high and low trustworthiness specifically because both represented endorsers with whom the recipients were familiar and reflect the use of this manipulation as it might have occurred in natural contexts. However, familiarity influences not only perceptions of trustworthiness, as intended, but other attributes, as well. For example, Tonya Harding and Nancy Kerrigan most likely differed in terms of likability, attractiveness, and even expectation of winning a medal at the Olympics. In fact, it may often be the case that naturally occurring trustworthiness will be associated with other factors, such as likability. Although unlikely, it is possible that one or even several of these other attributes could be responsibie for the results observed in Experiment 1.

Bases of trustworthiness. It can also be argued that individuals perceived Tonya Harding to be untrustworthy for unusual reasons that are not relevant to typical advertising situations. Tonya Harding was the recipient of extensive television and newspaper coverage suggesting that she was involved in an illegal attempt to maim her rival competitor. In contrast, trustworthiness for actual endorsers is more likely to be influenced by less sensational events. For example, advertising recipients might believe that a celebrity who endorsers many products cannot be believed because 
that celebrity will endorser any product, regardless of the product's merit. ${ }^{11}$ Although both Tonya and an over-endorsed celebrity may be perceived as untrustworthy, the basis of the trustworthiness may differ significantly. Tonya is perceived to be untrustworthy because of sensational events that are newsworthy, whereas an over-endorsed celebrity is perceived untrustworthy because of having endorsed too many products. If it is the sensational basis of Tonya's untrustworthiness that is responsible for the persuasion results of Experiment 1, than the implications for advertising are much more limited than if it is the untrustworthiness per se that is responsible for the persuasion results.

\section{EXPERIMENT 2}

We conducted Experiment 2 to replicate Experiment 1, while at the same time addressing the concerns that emerged from Experiment 1. In Experiment 2, we manipulated the information provided on unfamiliar endorsers to assure that these endorsers differed only on trustworthiness. In addition, the basis of their trustworthiness was manipulated to suggest that they were trustworthy or not as a result of over-endorsement. By manipulating trustworthiness in this manner, we addressed the concerns (a) that attributes other than trustworthiness were responsible for the results of Experiment 1 and (b) that the results of trustworthiness on persuasion found in Experiment 1 would generalize to trustworthiness as a function of over-endorsement. In addition, Experiment 2 assessed an indicator of the strength of the attitudes resulting from the trustworthy and untrustworthy endorsers-the accessibility of the attitude toward the endorsed brand. By assessing this indicator of attitude strength, we directly addressed the cogency of the two, quite different interpretations of the attitude interaction.

\section{Hypotheses}

As in Experiment 1, Experiment 2 manipulated Argument Quality and Endorser Trustworthiness. And as in Experiment 1, we hypothesized that an Endorser Trustworthiness $\times$ Argument Quality interaction would emerge on the measure of attitudes toward the product (H3), such that argument quality has a larger impact on peoples' attitudes when the endorser was low rather than high in trustworthiness (H3a). In addition, we hypothesized that attitudes resulting from a high and low trustworthy endorser would differ in terms of their bases (H4). Specifically, we hypothesized that the attitudes should be more highly correlated with the general positive perception of the endorser, as indicated by

\footnotetext{
${ }^{11}$ Such a belief has been used to explain why popular actors in the United States widely advertise in foreign markets, at the same time that they only selectively advertise in their home market.
}

the perception of trustworthiness, when the endorser was high rather than low in trustworthiness $(\mathrm{H} 4 \mathrm{a}) .{ }^{12}$ We hypothesized, also, that the attitudes formed as a result of the untrustworthy endorser would be more accessible (i.e., come to mind faster) than the attitudes formed as a result of the trustworthy endorser (H5). This configuration of results would provide strong support for the finding that untrustworthy endorsers engender not only greater elaboration than trustworthy endorsers, but also lead to stronger and more effective attitudes (i.e., the elaboration interpretation).

\section{Methods \\ Procedure}

A total of 106 undergraduate students at the University of Michigan participated. The students were randomly assigned to one of four experimental conditions in a $2 \times 2$ (Endorser Trustworthiness; High or Low $\times 2$ Argument Quality; Strong or Weak) factorial experiment. Experimental sessions were conducted in groups of 8 to 20 .

All participants received an experimental booklet in which the first page provided instructions. These instructions explained that they would read and evaluate three mock-ups of print advertisements. The instructions also explained that because these advertisements were still in development, for each advertisement they would read two sections-one section in which the spokesperson is described and a second section in which the wording for the advertisement is described. Participants were instructed to read the advertisements as if they came across them in a magazine and that they could spend as little or as much time reading each advertisement as they wished.

One advertisement was described on each of the second, third, and fourth pages of the booklet. For each advertisement, there was a section entitled "spokesperson" at the top half of the page and a section entitled "what the advertisement will say" at the bottom half of the page. The first ad was for a nutritional bar; the second ad was for a sports drink; and the third ad was the target, for Mercury InLine Skates. ${ }^{13}$ Immediately after reading the three advertisements, participants answered a series of questions on all three of the advertisements. At this time, participants provided their attitudes toward the product and their impressions of the endorser.

Participants then completed unrelated experimental material for approximately $30 \mathrm{~min}$. Following this unrelated material, all participants completed a computer task in which they responded to three attitude measures toward 20 different ob-

\footnotetext{
${ }^{12}$ Cognitive responses and an argument quality manipulation check were not assessed in Experiment 2 in that both had been collected and shown to be reliable in Experiment 1.

${ }^{13}$ The target advertisement was moved from the second to third advertisement position as a minor modification to assure that the results of Experiment 1 were not limited to specific advertising positioning.
} 
jects, including the target product (Mercury InLine Skates). ${ }^{14}$ This task assessed the accessibility of their attitude toward the product.

\section{Independent Variables}

Endorser trustworthiness. Endorser trustworthiness was manipulated by the information provided on the spokesperson. In all conditions, the spokesperson (Chad "Skates" McNamara) was described as likeable and expert. ${ }^{15}$ Specifically, all participants read that:

\begin{abstract}
A picture of Chad "Skates" McNamara doing a complicated InLine move with apparent ease dominates the ad. Chad is shown smiling and enjoying the Mercury InLine Skates. Chad is familiar to many InLine Skaters. He is considered the most likeable and friendly of all current top InLine Skaters. He is known as well for his laid back, friendly approach to life as he is for his moves on skates. He is considered to be one of the top InLine Skaters in the world, having won numerous free-style competitions in both national and international competition.
\end{abstract}

For participants assigned to the high trustworthiness conditions, Chad was additionally described as trustworthy. Specifically, the aforementioned information continued with:

One of the industry leaders said after learning that Chad would promote the Mercury InLine Skates, "there is no doubt that Chad knows InLine Skates, and InLiners will most definitely trust whatever he says, since he only puts his name on products that he believes in." Chad is without a doubt a top expert who truly knows InLine Skates. And the audience will be confident that he is telling them accurate information, because he is considered as trustworthy as he is expert and friendly. "That's Chad," said the industry leader, "friendly, expert, and trustworthy."

For participants assigned to the low trustworthiness conditions, Chad was additionally described as untrustworthy. Specifically, the aforementioned information continued with:

One of the industry leaders said after learning that Chad would promote the Mercury InLine Skates, "there is no doubt that Chad knows InLine Skates, but InLiners may not trust

\footnotetext{
${ }^{14}$ A 30-min interval was introduced before assessing attitude accessibility to provide a strong test of the predicted influence of elaboration on accessibility. A finding that elaboration resulted in differences in accessibility after 30 min filled with distracting material provides an even stronger case for the superiority of thoughtfully formed attitudes over nonthoughtfully formed attitudes.

${ }^{15}$ Recall that one of the purposes of Experiment 2 was to control for factors that may covary with naturally occurring trustworthiness, such as likeability. Thus, the fictitious endorser was described as likeable in both the trustworthy and untrustworthy conditions to isolate the influence of trustworthiness from these other possible factors.
}

whatever he says, since he puts his name on so many products, whether he believes in them or not." Chad is without a doubt a top expert who truly knows InLine Skates. But the audience may not be confident that he is telling them accurate information, because he is considered as untrustworthy as he is expert and friendly. "That's Chad," said the industry leader, "friendly, expert, and untrustworthy."

Argument quality. The strong and weak arguments used in Experiment 1 were modified to be gender appropriate (e.g., extremely large, rather than tiny, feet). Under the "what the advertisement will say" section, these arguments were presented, following a brief introduction paragraph and before a concluding line.

\section{Dependent Variables}

Following the three advertisements in the booklet were measures designed to assess attitudes and spokesperson perceptions. Participants completed three pages of measures-one page for each advertisement. On each page, the participants completed five items designed to measure attitude toward the advertised product and five items designed to measure perceptions of the spokesperson. Approximately 30 min after completing these measures, participants performed a computer task in which the response latency toward a variety of attitude objects was assessed. ${ }^{16}$

Manipulation check. Participants answered questions designed to serve as a manipulation check on the Endorser Trustworthiness manipulation. To assess perceived endorser trustworthiness, participants were instructed to "indicate your feelings about the spokesperson on the lines below." Imbedded within five endorser attributes was trustworthiness. Participants provided their rating of trustworthiness on an 9-point scale ranging from -4 (untrustworthy) to +4 (trustworthy).

Attitude measures. Participants answered five questions designed to assess their attitude toward the product. These questions were on 9-point scales ranging from -4 (negative, harmful, foolish, unfavorable, and bad) to +4 (positive, beneficial, wise, favorable, and good).

Attitude accessibility. Participants were given instructions that they would be presented with a series of questions on the computer screen and that they would respond by pressing one of two computer keys. Additionally, participants were instructed to respond both as quickly and as accurately as possible. Following these instructions, participants completed the response-time task, which consisted of 60 trials. The first 20 trials asked participants to respond to whether 20 attitude objects were good or bad. The second 20 trials asked participants to respond to whether the same 20 attitude ob-

\footnotetext{
${ }^{16}$ Dependent measures unrelated to the hypotheses of interest are not discussed further.
} 
jects were positive or negative, and the third 20 trials asked participants to respond to whether the attitude objects were favorable or unfavorable. The attitude objects included three automobiles (in Positions 1 through 3), the three sports-related products to which the participants were exposed (in Positions 4 through 6), and 14 unrelated consumer products (in Positions 7 through 20). Latency of the time between presentation of the attitude object and response to the attitudinal question was recorded by the computer. ${ }^{17}$

\section{Results}

\section{Trustworthiness Check}

The manipulation check for trustworthiness was subjected to a 2 (Endorser Trustworthiness $\times$ Argument Quality) analysis of variance. Not surprisingly, the endorser described as untrustworthy was perceived to be less trustworthy $(M=-1.4)$ than the endorser described as trustworthy $(M=$ 2.4), $F(1,102)=112, p<.0001 .18$

\section{Attitude Measures}

The five semantical differential items were averaged to create an attitude index. The standardized alpha coefficient for this index was .92 . The attitude index was analyzed by a 2 $\times 2$ (Endorser Trustworthiness $\times$ Argument Quality) analysis of variance. The means and standard deviations for the four experimental conditions are presented in Table 2 and graphed in Figure 2.

Of greatest importance, there emerged the predicted Endorser Trustworthiness $\times$ Argument Quality interaction, $F(1$, $102)=4.3, p<.05$-replicating the results of Experiment 1 and providing support for hypothesis H3. ${ }^{19}$ This interaction revealed that participants who read the advertisement endorsed by the untrustworthy endorser were more influenced by Argument Quality, $F(1,47)=34.4, p<.0001$, than were

\footnotetext{
${ }^{17}$ These instructions are based on Fazio (1990).

${ }^{18}$ In addition to this main effect for Endorser Trustworthiness, there was also a main effect for Argument Quality, revealing that endorsers who used strong arguments were perceived to be more trustworthy $(M=1.1)$ than endorsers who used weak arguments $(M=.1), F(3,102)=10.9, p<.01$. These two main effects were qualified by a significant Endorser Trustworthiness $\times$ Argument Quality interaction, $F(1,100)=6.6, p<.02$. This interaction revealed that the influence of Argument Quality on perception of Trustworthiness was greater when the endorser was untrustworthy $\left(M_{\text {strong }}=\right.$ $\left.-.4, M_{\text {weak }}=-2.2\right), F(1,47)=11.8, p<.002$ than when the endorser was trustworthy $\left(M_{\text {strong }}=2.4, M_{\text {weak }}=2.3\right), F(1,53)<1, p>.6$.

${ }^{19} \mathrm{As}$ in Experiment 1, analyses also revealed significant main effects for both Trustworthiness and Argument Quality. First, there was a significant main effect for Endorser Trustworthiness, $F(1,102)=7.4, p<.01$. Participants had more favorable attitudes toward the product when it was endorsed by the trustworthy spokesperson $(M=1.2)$ than when it was endorsed by the untrustworthy spokesperson $(M=.4)$. Second, there was a main effect for Argument Quality, $F(1,102)=27.7, p<.0001$. Not surprisingly, participants were more persuaded by the advertisement containing strong $(M=1.6)$ rather than weak $(M=.4)$ arguments.
}

TABLE 2

Means, Standard Deviations, and Cell Numbers for Attitude Index, Experiment

\begin{tabular}{lccccc}
\hline & \multicolumn{2}{c}{$\begin{array}{c}\text { Untrustworthy } \\
\text { Endorser }\end{array}$} & & & \multicolumn{2}{c}{$\begin{array}{c}\text { Trustworthy } \\
\text { Endorser }\end{array}$} \\
\cline { 2 - 3 } \cline { 5 - 6 } & $M$ & $S D$ & & $M$ & $S D$ \\
\hline Strong arguments & $1.5^{\mathrm{a}}$ & 0.8 & & $1.7^{\mathrm{b}}$ & 1.4 \\
Weak arguments & $-0.9^{\mathrm{c}}$ & 1.9 & & $0.7^{\mathrm{d}}$ & 2.0 \\
\hline
\end{tabular}

${ }^{\mathrm{a}} n=26 ;{ }^{\mathrm{b}} n=27 ; \mathrm{c}_{n}=23 ; \mathrm{d}_{n=28}$

participants who read the advertisement endorsed by the trustworthy endorser, $F(1,53)=3.8, p=.06$. Thus, the attitude indexes replicated the results of Experiment 1 and supported hypothesis $\mathrm{H} 3 \mathrm{a}$ - that an endorser of low trustworthiness can lead to greater scrutiny of product-related information than an endorser of high trustworthiness.

\section{Attitude Predictors}

As in Experiment 1, the attitudes for participants who read an advertisement comprised of strong arguments endorsed by the trustworthy spokesperson were as persuaded as the participants who read an advertisement endorsed by the untrustworthy spokesperson. However, the bases of these attitudes were predicted to differ. Specifically, Hypothesis $\mathrm{H} 4 \mathrm{a}$ predicted that the attitudes should be more highly correlated with the general positive perception of the endorser, as indicated by the perception of trustworthiness, when the endorser was high rather than low in trustworthiness. To examine this prediction, attitudes were correlated with the measure of perceived trustworthiness for each of the endorsers. The results revealed that the correlation between perceived endorser

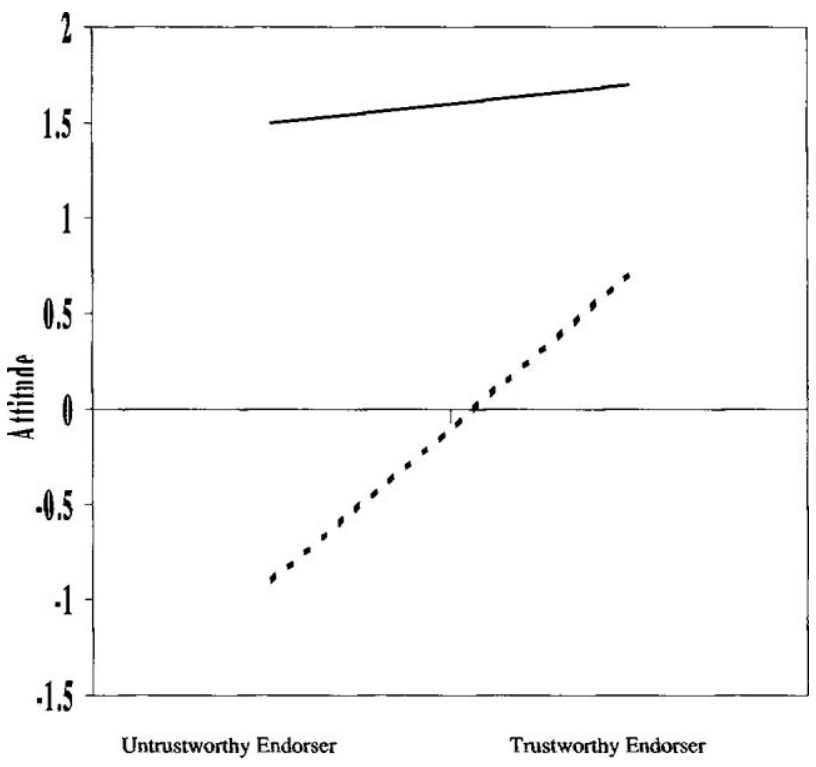

FIGURE 2 Attitude as a function of endorser trustworthiness and argument quality: Experiment 2. 
trustworthiness and the attitude index is $r=.67$ when the endorser is trustworthy and is $r=.46$ when the endorser is untrustworthy. To test whether the correlations differed significantly from each other, Fisher's $r$ to $z$ transformations were used. In support of Hypothesis $\mathrm{H} 4 \mathrm{a}$, attitudes were based marginally more on perceived trustworthiness when the endorser was trustworthy rather than untrustworthy, $z=1.6, p=$ .06 . This relation emerged even stronger when only the attitudes resulting from the strong arguments were considered, $z$ $=2.0, p<.03$. Specifically, the correlation between perception of endorser trustworthiness and attitude for the trustworthy endorser paired with strong arguments was $r=.60(p<$ $.001)$, whereas the correlation for the untrustworthy endorser paired with strong arguments was $r=.04(p>.8)$.

\section{Attitude Accessibility}

To test the critical hypothesis that the attitudes based on advertisements endorsed by the untrustworthy spokesperson would be more accessible than the attitudes based on advertisements endorsed by the trustworthy spokesperson (H5), the response latency data were analyzed in a manner recommended by Fazio (e.g., Fazio, Herr, \& Olney, 1984). Specifically, the response latency times were subjected to a reciprocal transformation and all data analyses were performed on these transformed scores. Mean latencies were calculated for the target attitude object (Mercury InLine Skates) by averaging the transformed latencies across the three different attitude measures. In addition, the mean latencies were calculated for the attitude object that immediately preceded the target attitude for each of the three trials. An analysis of variance revealed that response latencies to this nontarget attitude object did not vary as a function of Endorser Trustworthiness or Attitude Strength. Thus, the response latencies to this nontarget attitude object were used to control for individual differences in the latency of response to any attitudinal inquiry. Specifically, a response latency index was calculated by subtracting the target attitude response latencies from the nontarget attitude response latencies. This response latency index was subjected to a $2 \times$ (Endorser Trustworthiness $\times$ Argument Quality) analysis of variance. This analysis revealed only a significant main effect for Endorser Trustworthiness, $F(1,98)=4.6, p<.05 .{ }^{20} \mathrm{In}$ support of Hypothesis H5, participants who had read the advertisement endorsed by the untrustworthy spokesperson responded faster to the attitude questions than participants who f

\section{Conclusions}

Recall that we conducted Experiment 2 to address concerns that arose from Experiment 1. One concern had to do with the interpretation of the attitude interaction from Experiment 1. Given that there was no difference for trustworthiness for

\footnotetext{
${ }^{20}$ Because of computer difficulties, data for only 102 participants could be used in this analysis.
}

strong arguments, one could conclude that the untrustworthy endorser is not superior. In Experiment 2, a measure of attitude strength was collected. And this measure revealed that, even for the equivalently extreme attitudes associated with strong arguments, the attitudes formed from the advertisement endorsed by an untrustworthy endorser came to mind faster than the attitudes formed from the advertisement endorsed by the trustworthy endorser. Recall also that the attitude accessibility measure was collected $30 \mathrm{~min}$ after participants were exposed to the advertisements. Such a finding provides support for the elaboration interpretation of the attitude interaction.

Recall too that Experiment 1 used familiar endorsers. It is possible that the differences in product elaboration uncovered in Experiment 1 may have been the result of attributes upon which the endorsers differed other than trustworthiness. Experiment 2, in contrast, used endorsers with whom the participants were not familiar. Instead, the trustworthiness of the endorser was manipulated. And the results of Experiment 2 fully replicated the results of Experiment 1 . These findings suggest that trustworthiness can influence persuasion both when trustworthiness is associated with other factors (Experiment 1) and when trustworthiness is manipulated to be independent of these other factors. Finally, trustworthiness in Experiment 2 was manipulated by stating that an individual either endorsed too many products to be trusted or endorsed only products of merit. The results of Experiment 1 were replicated, even using this more naturalistic instantiation of trustworthiness. ${ }^{21}$

\section{DISCUSSION}

\section{Theoretical Insights}

\section{Multiple influences of trustworthiness}

This research is noteworthy in that it provides evidence for two distinct processes by which source trustworthiness can influence attitudes. First, source trustworthiness influenced the extent of elaboration. When source trustworthiness was low, recipients engaged in greater product-related elaboration than when source trustworthiness was high. Under low-source trustworthiness, argument quality had a larger impact on attitudes and product focused thoughts were more highly correlated with attitudes than when source trustworthiness was high. Second, source trustworthiness served as a simple cue when perceptions of high trustworthiness reduced message elaboration. That is, when the endorser was perceived as trustworthy, not only did this attenuate the impact of argument quality and message-based thinking, but attitudes tended to be influenced

\footnotetext{
${ }^{21}$ It is interesting to speculate whether the influence of endorser trustworthiness on persuasion generalizes to the trust with which individuals feel for companies. Future research should address this important question.
} 
by how trustworthy recipients perceived the source to be. ${ }^{22}$ Understanding when and how variables influence attitudes increases our understanding of the processes by which attitudes are changed-and the strength (effectiveness) of the resulting attitudes.

\section{Elaboration likelihood}

It is important to state explicitly the conditions under which the present experiments were run. The experiments were designed such that participants possessed the ability (i.e., they were not distracted nor were the ads presented in a capacity-straining manner) but did not necessarily possess the motivation to elaborate the ads. That is, the likelihood that participants would elaborate the information in the ads was not constrained to be either very high or low. Participants could choose to elaborate the ads or not, according to their motivation. As such, these experiments were conducted under moderate elaboration likelihood. Importantly, these are likely to be the precise conditions under which many recipients encounter advertisements. Had the elaboration likelihood level of this experiment been manipulated to be quite high, it is most likely that all participants would have been influenced by their thoughts in response to the advertisements (i.e., the argument quality manipulation) and the manipulation of endorser trustworthiness would not have had much influence on message elaboration, or would have influenced the nature of the elaborated thoughts. Similarly, had the elaboration likelihood been manipulated to be very low such as by distracting participants from elaborating (e.g., providing audiences with a television ad presented at a very rapid pace so that message processing was quite difficult; Moore, Hausknecht, \& Thamodaran, 1986; Smith \& Shaffer, 1995), it is most likely that the endorser trustworthiness manipulation would have served as a nonthoughtful message acceptance cue and there would have been little influence of the thoughts in response to the information (i.e., argument quality). Thus, under conditions of low elaboration likelihood, it is predicted that information provided by an untrustworthy endorser would be unthinkingly rejected.

\section{Strategic Insights}

Clearly, the question of when and why to use endorsers of high and low trustworthiness is a complicated issue that requires consideration of several factors. The point of this research is not to advocate for the use of untrustworthy endorsers. Rather, the point of this research is to suggest that

${ }^{22}$ This finding is consistent with the theoretical prediction of the multiple roles by which variables can influence attitudes advanced by the Elaboration Likelihood Model (ELM). Specifically, the ELM predicts that variables (such as trustworthiness) can influence attitudes by (a) simple cue effects, (b) increasing or decreasing elaboration, (c) serving as arguments as to the central merits of an attitude, and (d) influencing the valence of cognitive responses (i.e., biasing elaboration). under certain conditions, the use of an expert endorser about whom recipients have some suspicion may be advantageous, and to suggest that under certain conditions the use of a trustworthy source may be disadvantageous. In short, our experiment provides evidence that untrustworthy endorsers can lead to more elaborated attitudes and that trustworthy endorsers can lead to more nonthoughtful attitudes. This understanding of how endorser trustworthiness influences persuasion is particularly important given the relation between elaboration and attitude strength.

\section{When to use untrustworthy endorsers}

Our research suggests that untrustworthy (but expert) endorsers will be especially effective when two conditions are met. First and foremost, untrustworthy endorsers will be effective when presenting information that elicits positive PCRs when thoughtfully considered by the target of the advertisement. The advantage of using an endorser who is of questionable trustworthiness is that such an endorser will prompt increased elaboration of the information. Such increased elaboration will only be advantageous if the thoughts that arise in response to the information are positive (see Haugtvedt \& Priester, 1997). Under such conditions, the valence of the attitudes resulting from an untrustworthy endorser will be as, if not more, positive than the attitudes resulting from the use of a trustworthy endorser. Second, untrustworthy endorsers will be especially effective when the information is presented under conditions of moderate elaboration likelihood-when individuals have the requisite ability but lack the explicit motivation to elaborate the information conveyed in an advertisement. It is exactly under such conditions that the use of an untrustworthy endorser can increase the amount of thought that individuals expend on the product-related information. And it is this increase in elaboration that provides benefits that influence advertising effectiveness.

\section{Why to use untrustworthy endorsers}

Convergent evidence was provided in both studies that, although the attitudes resulting from the strong arguments were equally positive for both endorsers, the amount of thought upon which the attitudes were based differed significantly. And these differences in product-related thought are associated with important differences in attitude strength, as demonstrated by the differences in attitude accessibility revealed in Experiment 2. Given that the goal of advertising is to influence a consumer's behavior over a long period of time and in the face of counter-persuasion attempts by other advertisements, it is sensible for marketers to be interested in establishing thoughtful (i.e., elaborated) attitudes toward the product that are able to persist, resist, and guide behavior. And the use of an untrustworthy endorser might be a useful strategic tool in accomplishing this very goal. 


\section{ACKNOWLEDGMENTS}

This research was supported by National Institute of Mental Health traineeship T32 MH19728 to Joseph R. Priester and National Science Foundation Grants BNS 9021647 and SBR 9520854 to Richard E. Petty. This research was also funded by a grant from the University of Michigan Business School.

We thank the members of the Group for Attitudes and Persuasion at the Ohio State University for their helpful comments.

\section{REFERENCES}

Cacioppo, J. T., Harkins, S. G., \& Petty, R. E. (1981). The nature of attitudes and cognitive responses and their relationship to behavior. In R. E. Petty, T. M. Ostrom, \& T. C. Brock (Eds.), Cognitive responses in persuasion (pp. 31-54). Hillsdale, NJ: Lawrence Erlbaum Associates, Inc.

Cacioppo, J. T., \& Petty, R. E. (1982). The need for cognition. Journal of Personality and Social Psychology, 42, 116-131.

Cacioppo, J. T., Petty, R. E., Kao, C. F. and Rodriquez, R. (1986). Central and peripheral routes to persuasion: An individual difference perspective. Journal of Personality and Social Psychology, 51, 1032-1043.

Chaiken, S. (1980). Heuristic versus systematic information processing in the use of source versus message cues in persuasion. Journal of Personality and Social Psychology, 39, 752-766.

Chaiken, S., Liberman, A., \& Eagly, A. H. (1989). Heuristic and systematic processing within and beyond the persuasion context. In J. S. Uleman \& J. A. Bargh (Eds.), Unintended thought (pp. 212-252). New York: Guilford.

Chaiken, S., Wood, W., \& Eagly, A. (1996). Principles of persuasion. In. E. T. Higgins \& A. W. Kruglanski (Eds.), Social psychology: Handbook of basic principles (pp. 702-742). New York: Guilford.

Eagly, A. H., \& Chaiken, S. (1993). The psychology of attitudes. Fort Worth, TX: Harcourt Brace Jovanovich.

Elms, A. C. (1966). Influence of fantasy ability on attitude change through role-playing, Journal of Personality and Social Psychology, 4, 36-43.

Fabrigar, L. R., Priester, J. R., Petty, R. E., \& Wegener, D. T. (1998). The impact of attitude accessibility on the elaboration of persuasive messages. Personality and Social Psychology Bulletin, 24, 339-352.

Fazio, R. H. (1990). A practical guide to the use of response latency in social psychological research. In C. Hendrick \& M. S. Clark (Eds.), Review of personality and social psychology (Vol. 11, pp. 74-97). Newbury Park, CA: Sage.

Fazio, R. H. (1995). Attitudes as object-evaluation associations: Determinants, consequences, and correlates of attitude accessibility. In R. E. Petty \& J. A. Krosnick (Eds.), Attitude strength: Antecedents and consequences (pp. 247-282). Mahwah, NJ: Lawrence Erlbaum Associates, Inc.

Fazio, R. H., Herr, P. M., \& Olney, T. J. (1984). Attitude accessibility following a self-perception process. Journal of Personality and Social Psychology, 47, 277-286.

Greenwald, A. G. (1968). Cognitive learning, cognitive responding to persuasion, and attitude change. In A. G. Greenwald, T. C. Brock, \& T. M. Ostrom (Eds.), Psychological foundations of attitudes (pp. 147-170). New York: Academic.

Haugtvedt, C. P., \& Petty, R. E. (1992). Personality and persuasion: Need for cognition moderates the persistence and resistance of attitude changes. Journal of Personality and Social Psychology, 63, 308-319.

Haugtvedt, C. P., \& Priester, J. R. (1997).Conceptual and methodological issues in advertising effectiveness: An attitude strength perspective. In W. D. Wells (Ed.), Measuring advertising effectiveness: Advertising and consumer psychology (pp. 79-94). Mahwah, NJ: Lawrence Erlbaum Associates, Inc.

Haugtvedt, C. P., \& Wegener, D. T. (1994). Message order effects in persuasion: An attitude strength perspective. Journal of Consumer Research, 21, 205-218.
Hovland, C. I., Janis, I. L., \& Kelley, H. H. (1953). Communication and persuasion. New Haven, CT: Yale University Press.

Kallgren, C. A., \& Wood, W. (1986). Access to attitude relevant information in memory as a determinant of attitude-behavior consistency. Journal of Experimental Social Psychology, 22, 328-338.

Leippe, M. R., \& Elkin, R. A. (1987). When motives clash: Issue involvement and response involvement as determinants of persuasion. Journal of Personality and Social Psychology, 52, 269-278.

Mackie, D. M. (1987). Systematic and nonsystematic processing of majority and minority persuasive communications. Journal of Personality and Social Psychology, 50, 720-728.

Miller, N., \& Coleman, D.E. (1981). Methodological issues in analyzing the cognitive mediation of persuasion. In R. E. Petty, T. M. Ostrom, \& T. C Brock (Eds.), Cognitive responses in persuasion (pp. 105-126). Mahwah, NJ: Lawrence Erlbaum Associates, Inc.

Moore, D. L, Hausknecht, D., \& Thamodaran, K. (1986). Time compression, response opportunity, and persuasion. Journal of Consumer Research, 13, 85-99.

Petty, R. E., \& Cacioppo, J. T. (1979). Issue involvement can increase or decrease persuasion by enhancing message-relevant cognitive responses. Journal of Personality and Social Psychology, 37, 1915-1926.

Petty, R. E., \& Cacioppo, J. T. (1981). Attitudes and persuasion: Classic and contemporary approaches. Dubuque, IA: Brown.

Petty, R. E., \& Cacioppo, J. T. (1986). Communication and persuasion: Central and peripheral routes to attitude change. New York: Springer-Verlag.

Petty, R. E., Haugtvedt, C. P., \& Smith S. M. (1995). Elaboration as a determinant of attitude strength: Creating attitudes that are persistent, resistant, and predictive behavior. In R. E. Petty \& J. A. Krosnick (Eds.), Attitude strength: Antecedents and consequences. Mahwah, $\mathrm{NJ}$ : Lawrence Erlbaum Associates, Inc.

Petty, R. E., \& Krosnick, J. A. (1995). Attitude strength: Antecedents and consequences. Mahwah, NJ: Lawrence Erlbaum Associates, Inc.

Petty, R. E., Ostrom, T. M., \& Brock, T. C. (1981). Cognitive responses in persuasion. Hillsdale, $\mathrm{NJ}$ : Lawrence Erlbaum Associates, Inc.

Petty, R. E., Priester, J. R., \& Wegener, D. T. (1994). Cognitive processes in persuasion. In R. E. Petty \& J. A. Krosnick (Eds.), The handbook of social cognition (2nd ed., pp. 63-149). Hillsdale, NJ: Lawrence Erlbaum Associates, Inc.,

Petty, R. E., Schumann, D. W., Richman, S. A., \& Strathman, A. J. (1993). Positive mood and persuasion: Different roles for affect under high and low elaboration conditions. Journal of Personality and Social Psychology, 64, 5-20.

Petty, R. E., \& Wegener, D. T. (1997). Attitude change. In D. Gilbert, S. Fiske, \& G. Lindzey (Eds.), The handbook of social psychology (4th ed.). New York: McGraw-Hill.

Petty, R. E., Wegener, D. T., Fabrigar, L. R., Priester, J. R, \& Cacioppo, J. T. (1993). Conceptual and methodological issues in the Elaboration Likelihood Model of persuasion: A reply to the Michigan State critics. Communication Theory, 3, 336-363.

Petty, R. E., Wells, G. L., \& Brock, T. C. (1976). Distraction can enhance or reduce yielding to propaganda: Thought disruption versus effort justification. Journal of Personality and Social Psychology, 34, 874-884.

Priester, J. R., Nayakan Kuppam, D., Flemingm, M, A., \& Godek, J. (in press). The $\mathrm{A}^{2} \mathrm{SC}^{2}$ Model: The Influence of Attitudes and Attitude Strength on Consideration and Choice. The Journal of Consumer Research.

Priester, J. R., \& Fleming, M. E. (1997). Artifact or meaningful theoretical constructs?: Examining evidence for nonbelief- and belief-based attitude change processes. Journal of Consumer Psychology, 6, 67-76.

Priester, J. R., \& Petty, R. E. (1995). Source attribution and persuasion: Perceived honesty as a determinant of message scrutiny. Personality and Social Psychology Bulletin, 21, 637-654.

Rennier, G. A. (1988). The strength of the object-evaluation association, the attitude-behavior relationship, and the Elaboration Likelihood Model of persuasion. Unpublished doctoral dissertation, University of Missouri. 
Smith, S. M., \& Shaffer, D. R., (1995). Speed of speech and persuasion-Evidence for multiple effects. Personality and Social Psychology Bulletin, 21, 1051-1060.

Verplanken, B (1991). Persuasive communication of risk information: A test of cue versus message processing effects in a field experiment. Journal of Personality and Social Psychology, 17, 188-193.

Wright, P. L. (1973). The cognitive processes mediating acceptance of ad vertising. Journal of Marketing Research, 19, 53-62.

Wright, P. L. (1980). Message-evoked thoughts: Persuasion research using thought verbalizations. Journal of Consumer Research, 7, 151-175.

\section{APPENDIX}

\section{Strong Argument}

The most important reason to choose MERCURY ROLLER-BLADES is their fit: MERCURY ROLLER-BLADES are designed to fit any foot, no matter what size. They come in sizes 4 to 14 , with widths for extremely thin to extremely wide feet. And the specially designed cushioning makes it feel as if you are riding on air. Your feet feel free, which prevents them from tiring, and helps stabilize them. In addition to comfort, the MERCURY ROLLER-BLADES are designed with safety in mind. The excellent fit makes the MERCURY ROLLER-BLADES easier to control, which adds excitement. And control translates to fewer injuries. Consumer reports rated the MERCURY ROLLER-BLADES number one in safety for all roller-blades manufactured in 1993! And, the MERCURY ROLLER-BLADES come in every color from neon-yellow to midnight-blue, with 12 excit- ing colors in between. And if that's not enough, MERCURY ROLLER-BLADES are affordable. Check your local sports dealer and you will find that the MERCURY ROLLER-BLADES are, on average, $15 \%$ to $20 \%$ less expensive than other roller-blades.

\section{Weak Argument}

The most important reason to choose MERCURY ROLLER-BLADES is their fit: MERCURY ROLLER-BLADES are designed for very small and hard to fit feet, like mine. They come in sizes 4 to 6 , with widths for extremely thin feet. And the specially designed cushioning makes it feel as if you are riding on a fairly smooth concrete surface. Your feet feel heavy, which can make them tire quickly, but also stabilizes them. In addition to comfort, the MERCURY ROLLER-BLADES are designed with safety in mind. The extremely tight fit makes the MERCURY ROLLER-BLADES harder to control, which adds excitement. And control translates to fewer injuries. Consumer reports rated the MERCURY ROLLER-BLADES number six in safety for all roller-blades manufactured in 1993! And, the MERCURY ROLLER-BLADES come in every color from black to white, with 12 shades of grey in between. And if that's not enough, MERCURY ROLLER-BLADES are affordable. Check your local sports dealer and you will find that the MERCURY ROLLER-BLADES are, on average, only $15 \%$ to $20 \%$ more expensive than other roller-blades. 